\title{
Visual Space from Visual Motion: Turn Integration in Tethered Flying Drosophila
}

\author{
Reinhard Wolf and Martin Heisenberg ${ }^{1}$ \\ Theodor-Boveri-Institut für Biowissenschaften \\ Lehrstuhl für Genetik \\ D-97074 Würzburg, Germany
}

\begin{abstract}
Organisms navigating by path integration need to continuously measure their forward movement and their angular orientation with respect to an external reference. How they do it is little understood. Tethered flies at the flight simulator "navigate" in an artificial visual landscape without forward movement. They can return to a previously held orientation if the panorama provides a singularity (landmark) as reference. Surprisingly, in a regularly striped drum without singularities, they can use a temporal cue instead. In this experiment the arena is illuminated with only one color that is either green or blue. The arena is virtually divided into four quadrants. Whenever a quadrant boundary moves past an arbitrary point, the color of the arena light changes. When a fly is heated with one color it acquires a preference for the other one. Subsequently, it avoids the borders toward the potentially 'hot' quadrants even without touching them. The only way to achieve this is by turn integration, that is, by adding and subtracting all the turns it performs once it crosses the border. The color switch defining the border crossing resets the turn integrator, using the orientation of the arena at this moment as reference. In contrast, landmarks or, if it were available, the skylight compass enable the fly to establish by pattern learning any orientation as a reference. If the reference orientation coincides with the desired orientation, that is, if the animal stores the pattern while being oriented toward the goal, it can maintain its orientation without recourse to
\end{abstract}

${ }^{1}$ Corresponding author.

turn integration (which may be error prone).

\section{Introduction}

Returning to a point in space is a difficult orientation task that many animals must perform when returning to a fixed burrow or nest after foraging trips. The mechanisms by which they achieve this feat are little understood. The excursions on the way out, before food or prey are found, can be a tortuous path. The route back, however, should be as short as possible to secure the profit of the trip. Animals such as desert ants (Wehner et al. 1996) and honeybees (Menzel et al. 1996) meet this requirement with remarkable precision. Wherever they travel, they retain the direction and distance to their nest. In principle, they can derive this information from continuously monitoring the two parameters, orientation and distance travelled.

One detail of this task is to retrieve an angular orientation maintained earlier. To some extent, turning can be measured proprioceptively. Step sizes on the ground and angular acceleration detectors (e.g., halteres) in flight may provide relevant data. However, most animals with eyes rely on vision for precise control of orientation, as long as visual cues are available. The present study is concerned with the return problem only in the context of visual flight orientation, using the tethered fly Drosophila melanogaster at the flight simulator.

In free flight as well as in the flight simulator, the primary requirement in flight orientation is selfstabilization. Visual self-stabilization against rotations in the horizontal plane has been studied extensively in insects (for review, see Collett et al. 1993). In the flight simulator in which torque around the vertical body axis (yaw torque) is the only degree of freedom for visual control, Drosopbila adjusts its two flight motors to zero mean

$$
\begin{array}{lllllllllllllllll}
L & E & A & R & N & I & N & G & \begin{array}{l}
\mathbf{Z} \\
\mathbf{3 1 8}
\end{array} & M & E & M & O & R & Y
\end{array}
$$


yaw torque to provide a stable but slightly fluctuating orientation. During these periods of quasistraight flight, flies occasionally generate sudden large bursts of yaw torque, the so-called body saccades. These are the principal means of changing orientation (Heisenberg and Wolf 1979, 1984, 1993).

In earlier studies with the larger fly $\mathrm{Musca}$ domestica, Reichardt (1973; Reichardt and Poggio 1976) proposed that the vertical contours in the visual field determine the orientation of the flies in the flight simulator. This idea was based on the observation that the internal gain of the optomotor response was larger to motion from front to back than to motion from back to front, thus forcing the animal to head toward visual landmarks. It became clear, however, that the small fly Drosopbila can adopt and maintain any orientation with respect to landmarks in the flight simulator (Heisenberg and Wolf 1979) and that it is able to retrieve orientations to which it had been conditioned (Wolf and Heisenberg 1991).

When an animal uses vision to return to an angular orientation it had earlier maintained, it is generally thought to require an external reference such as the skylight compass or terrestrial landmarks. Here, we challenge this view using a regularly striped drum as the panorama in the flight simulator. Surrounded by this highly repetitive pattern, the fly cannot rely on landmark recognition for finding a particular orientation. This new experimental design reveals that Drosopbila continuously tracks its orientation by turn integration and uses an external event to reset this integrator.

\section{Materials and Methods}

Approximately 300 flies of wild-type Berlin were raised on $40-50 \mathrm{ml}$ of cornmeal-molasses standard fly food (for recipe, see Guo et al. 1996) in $180-\mathrm{ml}$ plastic vials at $25^{\circ} \mathrm{C}$ and $60 \%$ humidity and on a $16 \mathrm{hr}$ light/8 hr dark cycle. Only 3- to 4-dayold females were used for experimentation.

The flight simulator has been described before (Heisenberg and Wolf 1988). Briefly, the tethered fly is firmly attached to a torque meter and is positioned in the center of a circular arena that can be rotated by a fast motor. The angular velocity of the arena is made negatively proportional to the voltage signal of the torque meter. Thus, instead of rotating the fly in a stationary surround, the yaw torque rotates the panorama around the stationary fly (Fig. 1).

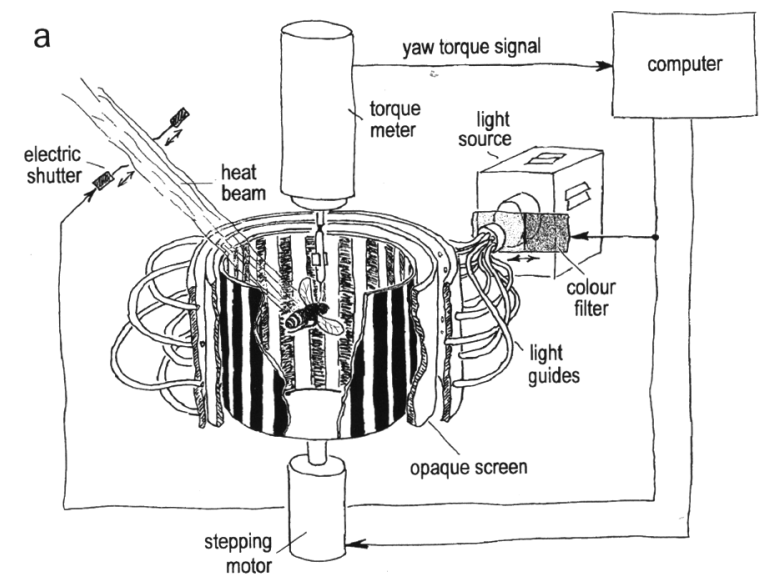

b

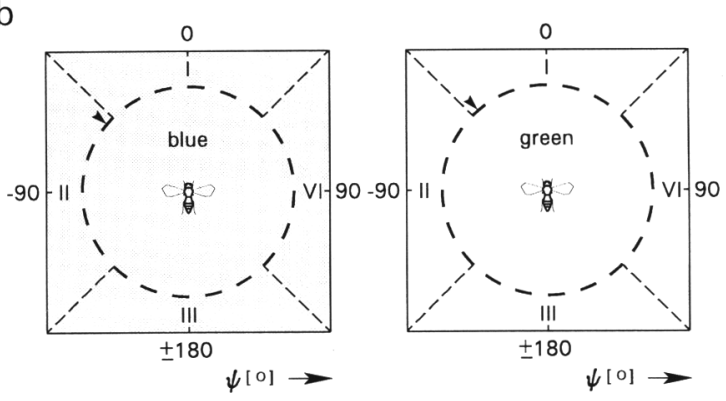

Figure 1: (a) Flight simulator with heat reinforcement. The switch of the color filters and the shutter intercepting the beam of infrared light (heat) are activated simultaneously by the computer at $90^{\circ}$ angular intervals of the arena. For details, see Materials and Methods. (b) The arena is a striped drum that is illuminated from behind either in blue or in green. The computer conceptually divides the visual field around the fly into four quadrants. If an arbitrary reference point on the drum (symbolized as an arrowhead) is in quadrants I $\left(-45^{\circ}<\Psi<45^{\circ}\right)$ and III $\left(\Psi>135^{\circ} ; \Psi<-135^{\circ}\right)$, the arena is illuminated in green; if the reference point is in one of the other quadrants $\left(-45^{\circ}>\Psi>-135^{\circ}\right.$ and $45^{\circ}<\Psi<135^{\circ}$ ), the arena light is blue. During training one of the colors is associated with heat.

The special procedure for color learning is outlined in Results. Pattern wavelength of the striped drum is $\lambda=18^{\circ}$; pattern contrast is about $m=0.95$ at a mean luminance of $I=10 \mathrm{~cd} / \mathrm{m}^{2}$ without color filters. For blue and green illumination of the arena, the light is passed through monochromatic broad band Kodak Wratten gelatin filters no. 47 (blue, cat. 1495787) and no. 99 (green, cat. 1496306), respectively. Filters can be exchanged by a fast servomotor (Faulhaber no. 3557/220) within 0.1 sec (Fig. 1).

Performance indices (PIs) were calculated from stored flight traces as $\mathrm{PI}=\left(t_{\mathrm{A}}-t_{\mathrm{B}}\right) /\left(t_{\mathrm{A}}+t_{\mathrm{B}}\right)$,

$$
\begin{array}{llllllllllllllll}
\hline & E & A & R & N & I & N & G & \begin{array}{l}
\mathcal{X} \\
319
\end{array} & M & E & M & O & R & Y
\end{array}
$$


with $t_{\mathrm{A}}$ and $t_{\mathrm{B}}$ being the total time spent in the 'cold' $\left(t_{\mathrm{A}}\right)$ and 'hot' $\left(t_{\mathrm{B}}\right)$ sectors during the respective 2-min periods of the training or memory test. Note that the PIs of the preference tests are calculated according to colors: $\mathrm{PI}_{\mathrm{pre}}=\left(t_{\mathrm{g}}-t_{\mathrm{bl}}\right) /$ $\left(t_{\mathrm{g}}+t_{\mathrm{bl}}\right)$, with $t_{\mathrm{g}}$ being the fraction of time spent in green light and $t_{\mathrm{bl}}$ the time in blue light. Error bars indicate S.E.M.s for the fluctuations among animals (n). Data were statistically tested against zero by $t$-tests unless otherwise indicated in the figure legends or text.

For the computer simulation in Figure 6, e and f (below) the 2-min torque trace of a preference test (single fly) was selected by the following criteria: The PI was close to zero, the fine structure of the yaw torque trace was similar under blue and green light, and the mean torque spike frequency was $\sim 0.5$ per sec. From this one torque trace, 13 artificial angular position traces were calculated using arbitrary starting postions $\psi(t=0)$. The simulated coupling coefficient was set to $-11^{\circ} / \mathrm{sec} /$ $10^{-10} \mathrm{Nm}$ (as in the real experiment) whenever the calculated angular position $\Psi(t)$ was in a cold quadrant (Fig. 6e) and to twice that value for $\Psi(t)$ in a 'hot' quadrant (Fig. 6f).

\section{Results}

In the present study, single flies were suspended in the flight simulator (Fig. 1a) in the center of a regularly striped drum such that the fly was able to maintain a stable relative orientation. The whole panorama was homogeneously illuminated from behind with one of two colors (blue or green) that were interchanged by a computer. In addition, the fly was instantaneously heated by a computercontrolled beam of near infrared (IR) light from a filtered microscope lamp. The heat served as a negative reinforcer because extended exposure is damaging to the fly.

The $360^{\circ}$ of the panorama were conceptually subdivided into four quadrants of $90^{\circ}$ (each with five black and five bright stripes). Whenever quadrants I or III were in front of the fly, heat was switched on and the arena was illuminated with one color. When quadrants II or IV moved to the front, heat was turned off and the arena light was switched to the other color (Fig. 1b). Note that at no time were the quadrants of the arena visible. It was only at certain fixed orientations of the arena that the temperature and the color (or only the color) of the panorama changed.
Under these conditions the fly avoided the sectors in which it was heated, and it still avoided them after heat was permanently switched off. The averaged results of 13 flies are shown in Figure $2 a$, and typical position traces of a "good learner" in Figure $2 \mathrm{~b}$. Avoidance of the heat-associated quad-
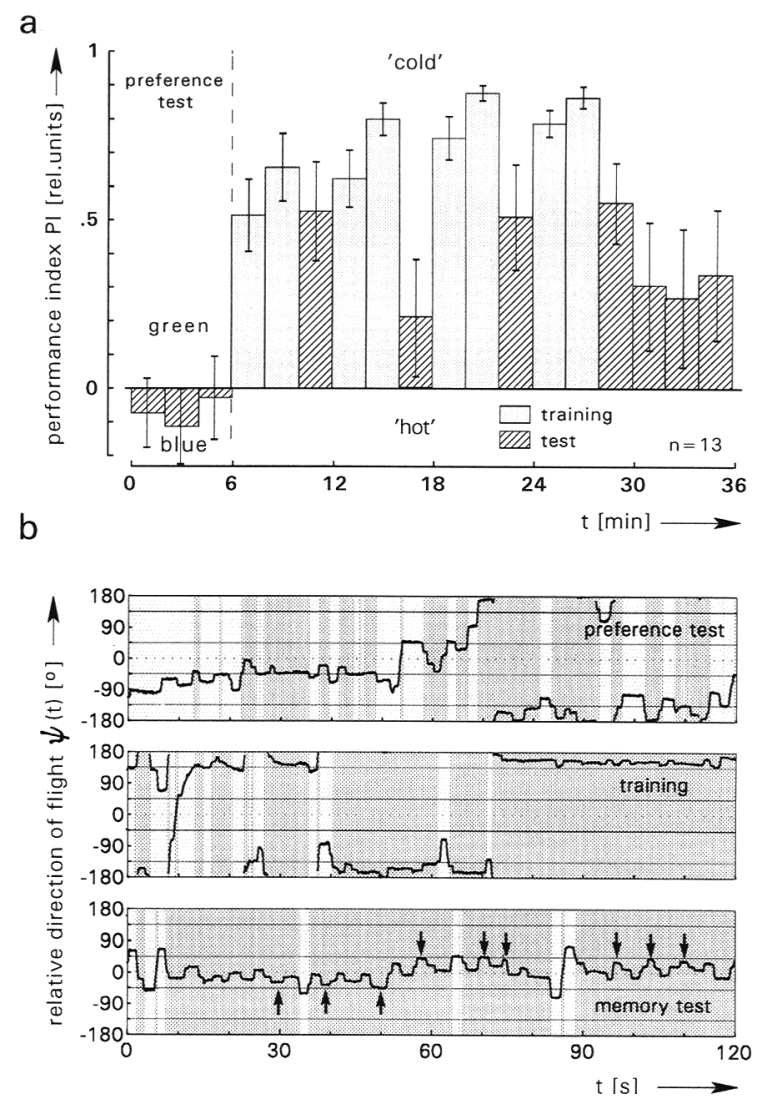

Figure 2: (a) Sequence of preference tests, training, and memory tests. Experimental time is divided into 2-min periods. Pls for training and memory tests are calculated as $\left(t_{\mathrm{A}}-t_{\mathrm{B}}\right) /\left(t_{\mathrm{A}}+t_{\mathrm{B}}\right)$, with $t_{\mathrm{B}}$ indicating the time the fly is exposed to the color combined with heat during training and $t_{\mathrm{A}}$ representing the time in the alternative color. PIs of the preference tests are calculated according to colors: $\mathrm{PI}_{\text {pre }}=\left(t_{\mathrm{g}}-t_{\mathrm{bl}}\right) /\left(t_{\mathrm{g}}+t_{\mathrm{bl}}\right) ; t_{\mathrm{g}}$ is the time spent in green light, and $t_{\mathrm{bl}}$ the time in blue light. Error bars are S.E.M.S for the number $(n)$ of flies. Pls of the first, third, and fourth memory test are highly significantly different from zero $(P<0.01)$. (b) Selected angular position traces of the reference point for 2-min flight periods before (top), during (middle), and after (bottom) training of one of the flies from the experiment of $a$. Light shading indicates the color associated with heat, whereas the shading represents the color combined with no heat. Note that only in the middle row was heat actually on during the marked periods. Arrows point to instances where the fly approaches the border without crossing it (cf. Fig. 6).

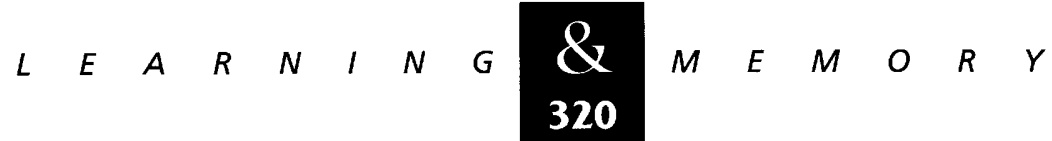


rants was about as good as in the standard experiment with four isolated patterns (Wolf and Heisenberg 1991) or a periodic random-dot texture (Dill et al. 1995). Some flies only required a few exposures to the heat to persistently stick to permissive pattern orientations (Fig. 2b). During the test periods (hatched bars in Figs. 2-5) in which quadrant boundaries were detectable only by the change in the overall color of the arena, the flies still showed a strong preference for those pattern orientations that were previously associated with no heat.

In the following experiment we asked whether this was a case of classical conditioning in which the flies associated one color with heat and the other with no heat or whether it was important that the flies had the opportunity to control the color and temperature switches as well as the rotations of the panorama themselves. We repeated the previous experiment but this time with only $2 \times 4 \mathrm{~min}$ of training. Avoidance and memory scores were comparable with those of the experiment in Figure 2a (Fig. 3a). This time, the exact position traces of the training periods of each fly were stored in the computer. Subsequently, these sequences of arena movements, heat and no-heat episodes and color changes were displayed to train naive flies in a second experiment. No learning was observed in this open-loop situation (Fig. 3b). Apparently, it was important for the success of the training that the flies were in command of the arena movements (operant conditioning).

In the operant training above, flies controlled not only the movement of the drum but indirectly also the temperature and color switches. To dissociate these two components of the operant activity, a further variant of the experiment was performed (Fig. 4). The operant control of orientation in the flight simulator was combined with a classical conditioning procedure. The fly's yaw torque determined the angular velocity of the drum, and one of the colors was presented with heat, the other with no heat. Now, however, the two color/ temperature conditions were not linked to quadrants. They were switched by the computer at arbitrary orientations of the arena for varying lengths of time. Under these conditions, the mean memory score was significantly positive $(P<0.01)$, indicating that flies can be classically conditioned to associate heat and colors. However, learning scores are considerably smaller than after operant conditioning. On average, in the new experiment a fly was heated for $25 \%$ of the time, which was $\sim 10 \%$ more than in the operant and replay experiments
(Figs. 2 and 3). Presumably, the small learning effect would have been further diminished if heat had been on for only $15 \%$ of the time, as above.

In the experiments of Figures 2, 3a, and 4, flies must have acquired a preference for the quality of the arena light associated with the permissive temperature or an aversion for the alternative illumination condition or both, because the periodic grating in the flight simulator had no singularities that might have been used as a reference for selecting certain orientations. Probably, the flies relied on hue discrimination. Color discrimination
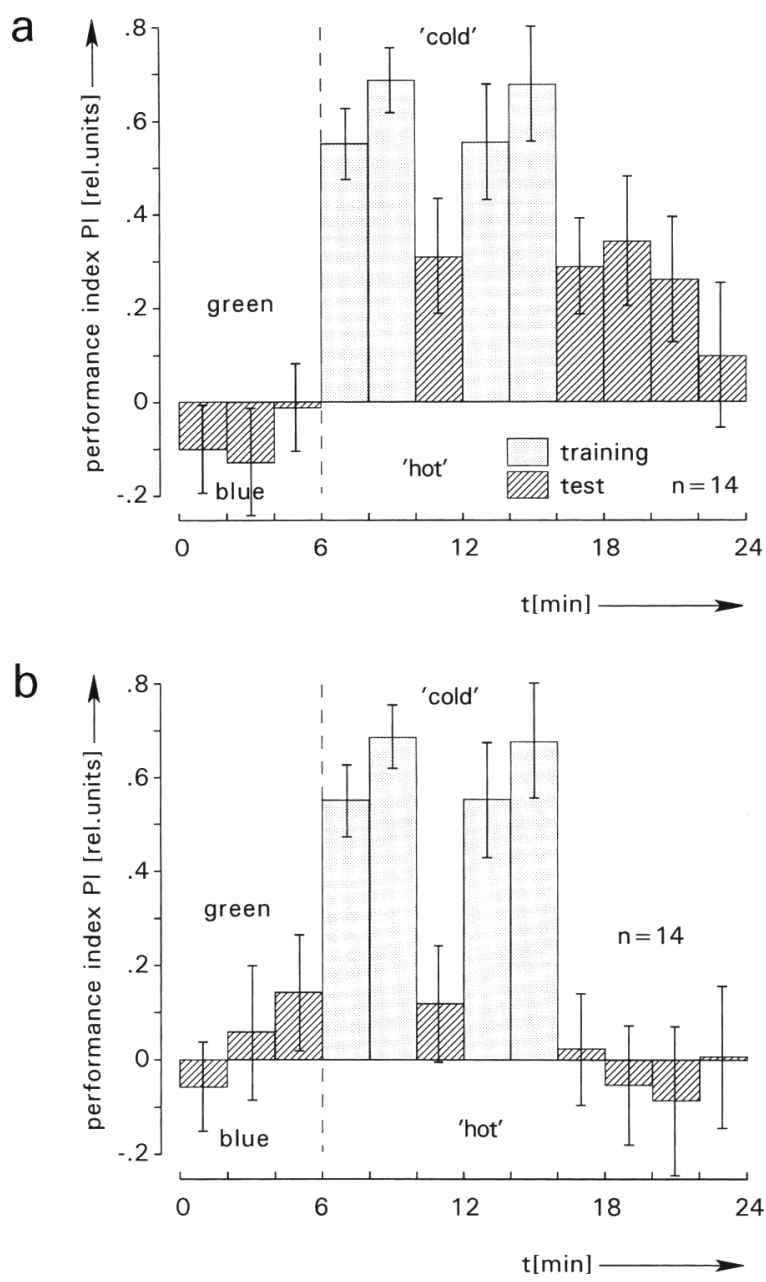

Figure 3: Master/replay experiment. (a) Flies were trained as in Fig. 2 except that only two training periods were applied. During training the position trace of the striped drum was digitally recorded with an accuracy of $\left(\delta<<0.1^{\circ}\right.$ and was played back to a naive fly with the same color changes and heating periods. Training under open-loop conditions had no learning effect. Error bars are S.E.M.S for $n$ flies.

$$
\begin{array}{llllllllllllllll}
\hline & E & A & R & N & I & N & G & \underset{321}{\mathbf{Z}} & M & E & M & O & R & Y
\end{array}
$$




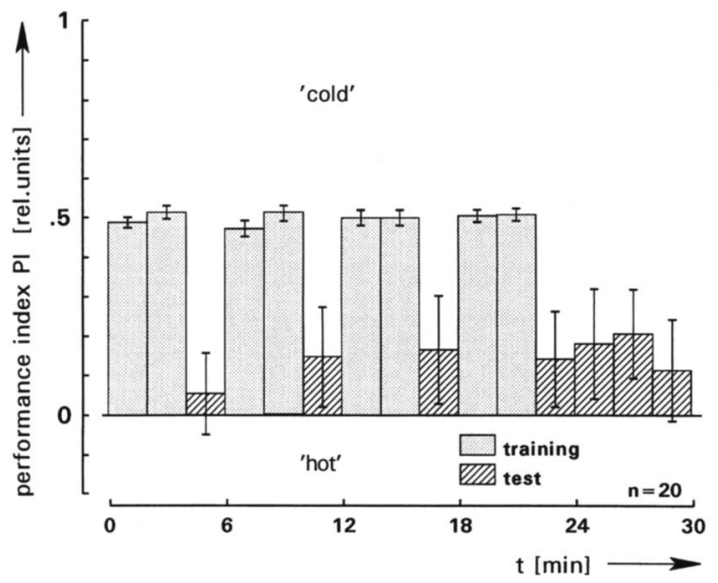

Figure 4: Classical conditioning in the flight simulator mode. The fly controlled the angular velocity of the striped drum (flight simulator), but the computer switched between the two colors and temperatures at arbitrary times (cf. dwelling times in Fig. 5). Overall time in the heat $\left(t_{\mathrm{B}}\right)$ was $25 \%$ of each 2 -min period. Individual periods in the heat (i.e., artificial "dwelling times") ranged from $0.1 \mathrm{sec}$ to $3.25 \mathrm{sec}$ and had a flat probability distribution. The distribution of periods at the low temperature was equally flat ranging from 0.1 to $9.82 \mathrm{sec}$. Taken together, the seven Pls of the memory tests are significantly $(P<0.01 ; t=3.06$; one sample $t$ test) different from zero. Note that in this experiment we omitted the color preference test because we considered the possibility that presenting the same color first without and then with heat might confuse the animals. Error bars are S.E.M.s for $n$ flies.

learning has been studied in Drosopbila earlier (Menne and Spatz 1977; Hernandez de Salomon and Spatz 1983). To test this supposition, we applied in a pilot experiment two intensities [standard intensity and 30\%; a simultaneous pattern contrast of $30 \%$ is a strong optomotor stimulus (Götz 1964)] of white light instead of two colors. Not only could the flies not learn under these conditions, they did not even properly avoid the heat during training, and several of them were damaged by excess heat (data not shown). If the flies are indeed unable to discriminate the two qualities of the arena light by their intensities, they must rely on their hues. Although this issue is not resolved, we will, for simplicity, refer in the following to the two qualities of the arena light as the two colors (green and blue). However, we are not trying to prove color discrimination learning here.

Our main interest in this study lies in orientation. Previously, it was demonstrated by measuring dwelling times that in an arena with random-dot textures the flies develop a lasting preference for the no-heat condition but seem not to avoid the heat-associated patterns in the memory test (Dill et al. 1995). In Figure 5 we calculated dwelling times for the present learning experiment. During training (shaded bars), the flies shortened their mean dwelling time in the heated sectors compared with the pretest period without heat. In the 'cold' sectors, mean dwelling time went up during training, indicating that flies developed a strategy to avoid crossing the quadrant border into the 'hot' sectors. In the memory test, both mean dwelling times, the one in the 'cold' and the one in the 'hot' sector, relaxed slightly with respect to the training period but were still significantly different from the pretest period $(P<0.05$ that dwelling times during the memory test are not smaller than during pretest). Thus, in contrast to the experiment using random-

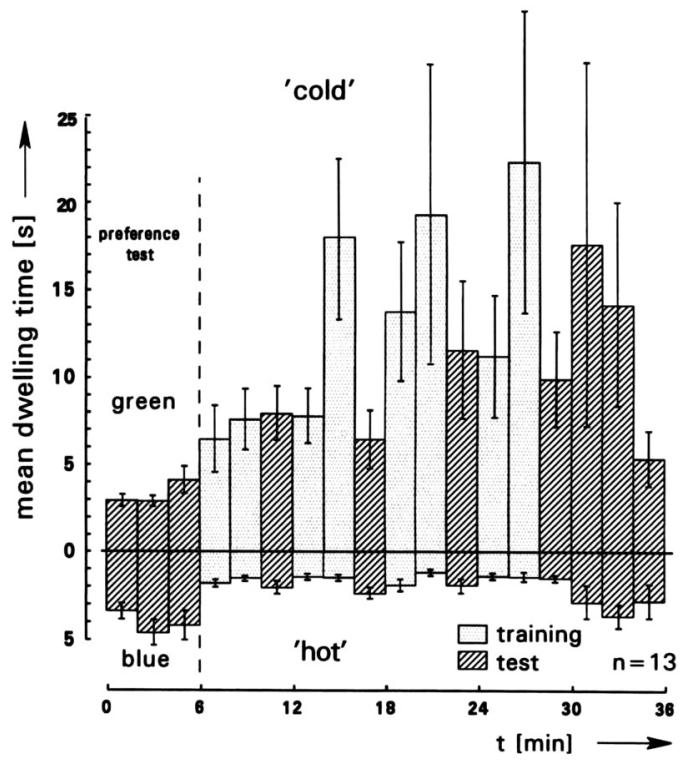

Figure 5: Mean dwelling times (i.e., the time a fly spends in one quadrant) in both the 'cold' and 'hot' quadrants of the experiment in Fig. 2. The dwelling times in the 'hot' quadrants were significantly shorter in the memory test immediately following the training than the corresponding dwelling times of the preference test $(P<0.005$; alternate $t$-test for Gaussian populations with different S.D.s; Welch's approximate $t=4.777$ ). This indicates that the flies associated not only the corresponding color with the permissive temperature but also the other one with heat. Note that in this case medians were calculated for each fly instead of mean values. Shown are the means of the medians of 13 flies $(n)$. Error bars are S.E.M.S of the medians. Statistical relevance was evaluated by Wilcoxon (paired) and $u$-tests (unpaired) (Dill et al. 1995).

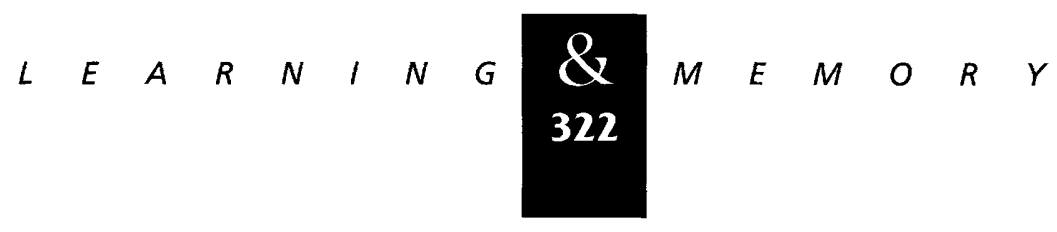


dot textures (Dill et al. 1995), both the permissive $\left(\mathrm{CS}_{\text {cold }}\right)$ and the nonpermissive $\left(\mathrm{CS}_{\text {hot }}\right)$ cues are memorized. Heat induces an aversion, whereas no heat leads to a preference for the respective color.

Remarkably, the flies express their preference and aversion for the respective color during the memory test by their spatial orientation preference. How can they do that? They have no landmarks and no spatial color contrast to distinguish the quadrants in the regularly striped drum. One way the flies could prolong the periods of the attractive color and shorten those of the dreaded one would be to regulate the frequency of turning maneuvers. The fly would avoid turning as soon as it noticed the attractive color and would increase its general turning activity if the color changed to the disliked one. This is not how the fly does it. Although overall turning activity is higher in the avoided color than in the preferred one, this effect disappears if one corrects for the difference in dwelling times. For example, there is no difference in the turning activity during 3-sec episodes in either temperature quadrant (data not shown).

If the fly does not regulate its amount of turning to stay longer in the 'cold' and shorter in the 'hot' quadrants, the only alternative is that it controls its direction of turning. To do this in a meaningful way, the fly needs information about its an- gular position relative to the quadrant border. In principle, the fly could register the orientation of the panorama at the moment the color switches and then measure and add its subsequent turns. This procedure could tell the fly at any moment how far its orientation deviated from the border it had last crossed. With this information the fly could direct its turns toward and across the border if it were in the heat-associated quadrant and away from the border in the 'cold' one. We show in the following that this is indeed the mechanism the fly uses.

The angular positions of the flies with respect to the quadrant border were plotted as a histogram (Fig. 6). The border was arbitrarily defined as $\Psi=0^{\circ}$. All four borders with their adjacent $\Psi=+$ / $-45^{\circ}$ sectors were superimposed. Sectors not heated during training are always shown to the left of the border $\left(\Psi<0^{\circ}\right)$, and heated ones to the right $\left(\Psi>0^{\circ}\right)$, regardless of their location and orientation in the panorama. The same arrangement was used for the preference and the memory tests in which no heat was involved (Fig. 6a,c,d).

As expected, the histogram of the preference test is flat (Fig. 6a). The quadrant borders were superimposed according to heat/no heat during training and not according to color. Therefore, a spontaneous color preference would not show

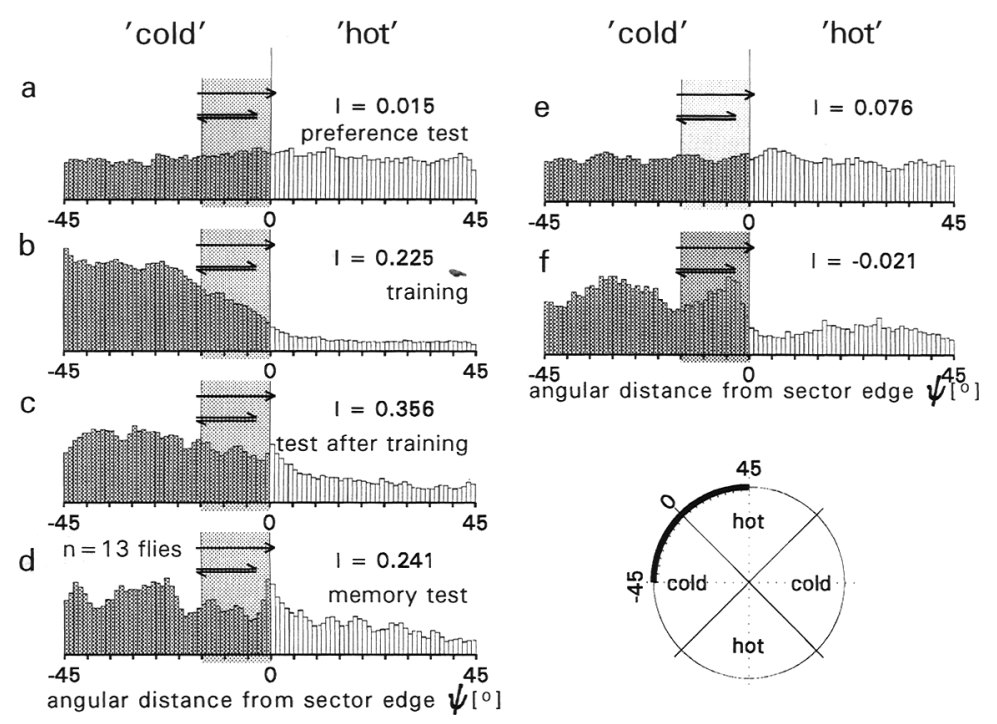

Figure 6: $(a-d)$ Histograms of the flies' orientations in relation to the virtual quadrant boundaries of the striped drum. The bin width of the histograms was $1^{\circ}$. All boundaries were superimposed, as schematically indicated by the inset at lower right. The border zone, a $15^{\circ}$ sector in the 'cold' quadrant (dark shading) adjacent to the boundary, was marked in light shading. It served to define the border-avoidance index (I), which was defined as $\mathrm{I}=\left(\mathrm{E}_{\mathrm{C}}-\mathrm{E}_{\mathrm{H}}\right) /\left(\mathrm{E}_{\mathrm{C}}+\mathrm{E}_{\mathrm{H}}\right)$, with $\mathrm{E}_{\mathrm{C}}$ indicating the number of exits from the border zone into the 'cold' quadrant and $\mathrm{E}_{\mathrm{H}}$ the number of exits into the 'hot' quadrant. As explained in the text the I value depends on the width of the border zone. An I value close to zero was chosen for the preference test. This implies that a fly entering the $15^{\circ}$ sector adjacent to the border (border zone) from the center of the 'cold'

quadrant leaves it about equally often on either side. In contrast, the conditioned fly leaves this sector about twice as often by turning toward the center of the quadrant rather than into the other quadrant. $(e, f)$ Thirteen artificial orientation histograms were calculated from a single 2-min yaw torque recording using different starting positions $[\psi(t=0)]$, and were superimposed as described above. In $f$ a twofold modulation of yaw torque in the heat was assumed. No significantly positive border-avoidance index was generated (for more details, see Materials and Methods and text). Data are from the experiment in Fig. 2.

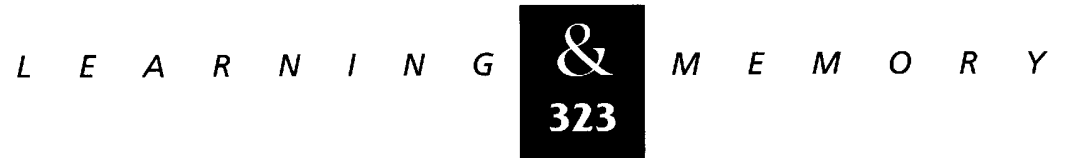


even if it existed, because 6 of the 13 flies were conditioned to associate heat with blue; the other seven with green. We have evaluated the data for color preference (Figs. 2a and 3a,b). Although individual flies can be strongly biased to one color, the whole group does not show a significant preference.

During training there was an expected low probability for orientations toward the heat-associated sectors and a significantly higher probability for no-heat orientations (Fig. 6b). The linear decline in probability between $\Psi=-20^{\circ}$ and $\Psi=5^{\circ}$ suggests that flies in the 'cold' quadrant avoid getting too close to the border. However, this distribution of orientations does not prove that the fly controls its direction of turning. Using the yaw torque data of a single fly from a 2 -min preference test of the experiment shown in Figure 2a, we calculated 13 artificial angular position traces using arbitrary starting positions. From these data we obtained an artificial orientation histogram (Fig. 6e). Taking yaw torque in the 'hot' sectors to be twice that in the 'cold' ones and calculating again 13 position traces, we obtained the histogram shown in Figure 6f. In this model, calculation of the strong "preference" for the 'cold' sector is solely owing to the temperature dependence of the yaw torque modulations. No directional control of turning is introduced. The artificial histogram in Figure $6 \mathrm{f}$ has a narrower transition range between the high and low probabilities in the cold and hot quadrants ( $\Psi=-5^{\circ}$ to $\Psi=3^{\circ}$ ) than the real one measured during training (Fig. 6b), but in the model calculation, the transition range could probably be extended by adjusting some of the parameters in the yaw torque trace and taking into account a slight delay in the effect of the heat.

To obtain direct evidence that the fly controls turning polarity, its behavior near the border was investigated in more detail. It was asked whether in the 'cold' quadrant a conditioned fly approaching the quadrant border has indeed a higher probability than a naive fly to turn away from the boundary. We again used the data of Figure $2 a$ to calculate a so-called border-avoidance index (I). A "border zone" was defined in the 'cold' quadrant between $\Psi=-15^{\circ}$ and $\Psi=0^{\circ}$. Whenever the fly entered the border zone from the 'cold' side, we determined to which side it left. When it triggered the color switch, this was scored as a bot side exit; when it touched again the $\Psi=-15^{\circ}$ border, this was taken as cold side exit. The border-avoidance index $I$ is defined as the difference of 'cold' side exits and 'hot' side exits divided by the sum of all exits.

The I value is dependent on the width of the border zone that was deliberately chosen to give a small I value in the preference test. Thus, $\mathrm{I}=0.015$ in the preference test. This indicates the fly leaves the border zone about equally often on either side when entering from $\Psi=-15^{\circ}$. During training (Fig. 6b), the border-avoidance index goes up to $\mathrm{I}=0.225$. Interestingly, during the memory tests in the first 2 min after the training (Fig. 6c), the border-avoidance index goes up even further $(I=0.356)$ and is still $I=0.241$ from 2 to $8 \mathrm{~min}$ after training (Fig. 6d). In other words, after entering the border zone from the center of the 'cold' quadrant, the conditioned fly turns about twice as often toward the center of the quadrant than into the other quadrant. In the model calculation (Fig. $6 e, f)$ using temperature-dependent differences in turning activity, the I value does not go up. We, therefore, interpret the high border-avoidance indices in Figure $6 b-d$, to indicate that the conditioned fly has information about the angular position of the quadrant border without touching it (see also arrows in Fig. 2b).

\section{Discussion}

We have shown in this study that the fly in the artificial environment of the flight simulator can take the orientation of the periodic grating at the moment of the color switch as a reference from which to measure its actual angular deviation. Using this information it can then direct its turns away from and presumably also toward and across this reference orientation depending on whether it is in the 'cold'/color A or 'hot' / color B environment.

Our experimental setup for color discrimination learning is particularly valuable because it dissociates turn integration from landmark recognition. In the drum with 20 identical black and white stripes, the fly has no landmarks by which to distinguish the 'hot' and 'cold' quadrants. It needs the vertical visual contours only for optomotor control (i.e., for flying straight) and for measuring the size of its turns (see below). In addition, it requires an integrator that continuously adds up the turns and is reset to zero by the color change. The integrator uses the pattern orientation at the moment of the reset as its zero reference.

We do not yet know how the fly measures its

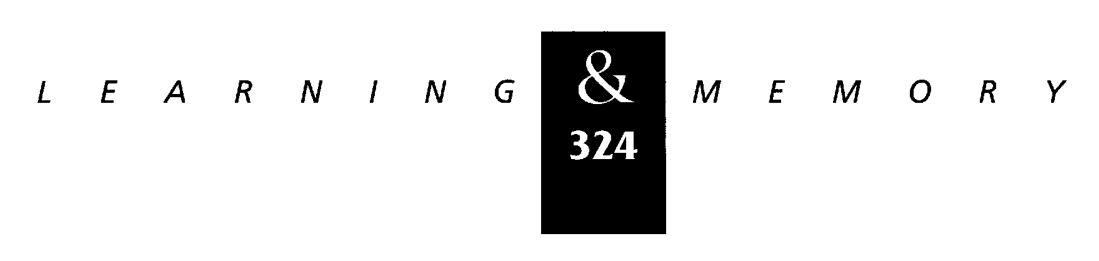


turns. In principle, it could integrate the efference copies of the turning maneuvers if it had previously calibrated the efficacy of its yaw torque in eliciting left and right turns of the drum in the flight simulator. We have shown previously ( $R$. Wolf and M. Heisenberg, unpubl.) that Drosophila can adapt very well to different coupling coefficients for the two directions of turning, implying that independent calibration of the two directions may be required. In the special case of the striped drum, the fly cannot obtain an absolute calibration. However, it can (and must) adjust the relative efficacy of yaw torque to the left and yaw torque to the right.

Alternatively or in addition, the fly may integrate the output of its movement detectors. The drawback of using motion detectors is that their output may depend on angular velocity as well as amount of contour and pattern contrast in the visual field. They also require calibration that might be partially provided phylogenetically. If the movement detector signal could be used for measuring turns and had an intrinsic calibration, it could also be used to calibrate the efference copies of turning maneuvers. It will be interesting to investigate how well fly movement detectors can measure turn size.

For unknown reasons, this avoidance learning by turn integration seems not to work with intensity instead of color changes. Either the turn integrator is not triggered and reset by the intensity change or the fly quickly adapts, thus finding it difficult to firmly assign a positive or negative value to either light intensity. This question needs to be pursued with different intensities and spectral compositions of the "white" light.
Most visual environments contain singularities (landmarks or the skylight compass). Thus, patterns and pattern recognition are used with turn integration. But, what are they good for? As alluded to above, patterns with singularities enable the fly to calibrate the efference copies of its turns. To achieve this, the fly must determine how much yaw torque is required for full $360^{\circ}$ turns in either direction. The fly stores the landmark pattern retinotopically and at the same time resets the turn integrator. Then, it generates a series of body saccades in one direction until the actual retinal image again matches the memory template. The match tells the fly it has performed a $360^{\circ}$ turn. Finally, it repeats the procedure for the other direction of turning. We do not know whether insects have to rely on this procedure.

The main advantage of pattern recognition (landmark learning) for angular orientation is probably different. In the present experiment the fly is forced to use the orientation of the drum as external reference at the moment of the color switch. In contrast, with landmark recognition (see, e.g., Dill et al. 1995) it can establish a new reference orientation with each new "snapshot" it takes of the panorama. This constitutes an invaluable degree of freedom because the fly can now optimally position the reference (see below).

Earlier experiments showed that the flies developed a preference for the center of the 'cold' quadrant if the arena wall was decorated with periodic random-dot textures (Fig. $7 b$; Dill et al. 1995). This suggested that the flies tried to stay as far away as possible from the 'hot' quadrants. Why should the flies adopt a different strategy in the present experiment? However, the orientation his-
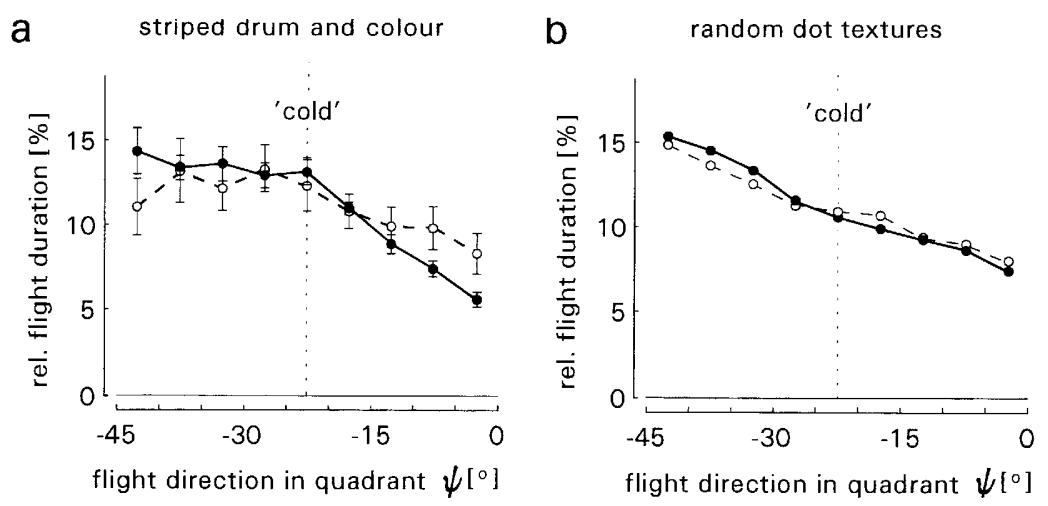

Figure 7: Orientation by turn integration but without landmark memory is less accurate than orientation using both. Orientation histograms of color avoidance learning (a) (data from the experiment in Fig. 2) are compared with the corresponding histograms obtained in pattern avoidance learning with periodic random dot textures (b) (data from Dill et al. 1995). Orientation histograms are shown only for 'cold' quadrants (note that histograms in a correspond to left parts of Fig. 6 b,c). In a the probability distribution in the center of the quad rant $\left(-22.5^{\circ}>\Psi>-45^{\circ}\right)$ is flat $(P=0.09$ and $P=0.44$ for a difference from a horizontal line), whereas in $b$ it is significantly different from horizontal $(P<0.001$ and $P<0.005)$ indicating that the flies try to stay as far away from the quadrant borders as possible. (O) Training; (O) memory tests immediately after training.

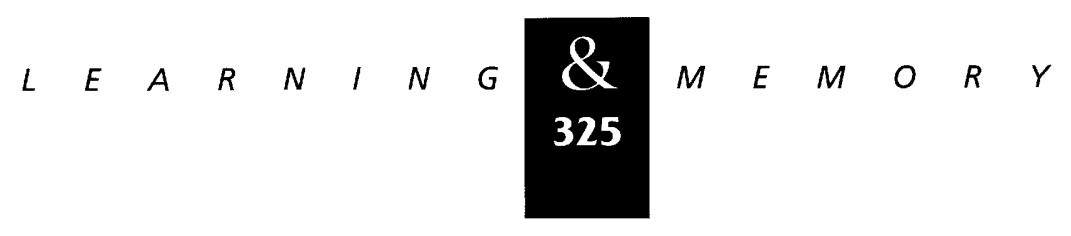


tograms are flat in the middle of the quadrant (around $\Psi=-45^{\circ}$; Fig. 7 a). We propose that turn integration without landmark recognition does not allow the fly to establish a preferred orientation in the center of the 'cold' quadrant. Probably, the turn integrator loses accuracy with time after being reset and is much less accurate at high values than close to zero. In other words, it may be difficult to navigate for a long time at a large angle relative to the reference orientation.

Probably, in the random-dot arena the fly starts out the same as in the color discrimination experiment. It establishes an external reference at the moment of the temperature switch by taking a snapshot of the panorama. While training proceeds, however, it can gradually establish new reference orientations closer to the optimal angular position in the center of the 'cold' quadrant. One way to find the central orientation would be by trial and error elimination, but the brain might also have the means to measure the center of the quadrant and to rotate the memory image accordingly.

Rotation of a memorized orientation reference occurs when bees use the skylight compass to choose the same flight direction at different times of day. Moreover, relying on path integration to return straight home from a foraging trip, a bee must have stored a memory image as a reference. When this is matched by the actual image, then the bee is heading toward the hive (the home vector). If a bee travels in a direction that differs from the home vector, the bee must rotate the home vector en route, taking into account the current travel direction, speed, and distance from the hive (Müller and Wehner 1988; Hartmann and Wehner 1995). It is not known whether a bee can continuously adjust the home vector during the whole trip by gradually rotating it "mentally" or whether it must occasionally take new snapshots to establish a new reference of the actual home vector orientation.

Our new paradigm here demonstrates that independent of landmark learning, insects are able to visually measure and integrate their flight turns. They can measure how far their actual course deviates from a reference orientation. Landmark learning in angular orientation then adds the ability to deliberately reposition the external reference. Comparison of the new and old data (Fig. 7) suggests that this happens in the flight simulator during landmark learning. Whether Drosopbila, like bees and ants, are able to rotate the reference according to some other neuronal process (e.g., for using the skylight compass at different times of the day or for path integration) is not known.

\section{Acknowledgments}

We thank R. Menzel and K.G. Götz for stimulating comments on the manuscript and T. Zars for language editing. The work was supported by grants of the Deutsche Forschungsgemeinschaft (He 986) and the Fonds der Chemischen Industrie.

The publication costs of this article were defrayed in part by payment of page charges. This article must therefore be hereby marked "advertisement" in accordance with 18 USC section 1734 solely to indicate this fact.

\section{References}

Collett, T., H.-O. Nalbach, and H. Wagner. 1993. Visual stabilization in arthropods. In Visual motion and its role in the stabilization of gaze (ed. F.A. Miles and J. Wallmann), pp. 239-263. Elsevier, Amsterdam, Netherlands.

Dill, M., R. Wolf, and M. Heisenberg. 1995. Behavioral analysis of Drosophila landmark learning in the flight simulator. Learn. \& Mem. 2: 152-160.

Götz, K.G. 1964. Optomotorische Untersuchung des visuellen Systems einger Augenmutanten der Fruchtfliege Drosophila. Kybernetik 2: 77-92.

Guo, A., L. Liu, S.-Z. Xia, C.-H. Feng, R. Wolf, and M. Heisenberg. 1996. Conditioned visual flight orientation in Drosophila: Dependence on age, practice and diet. Learn. \& Mem. 3: 49-59.

Hartmann, G. and R. Wehner. 1995. The ant's path integration system: A neural architecture. Biol. Cybern. 73: 483-497.

Heisenberg, M. and R. Wolf. 1979. On the fine structure of yaw torque in visual flight orientation of Drosophila melanogaster. J. Comp. Physiol. A 130: 113-130.

1984. Vision in Drosophila. In Studies of brain function, Vol. XII (ed. V. Braitenberg). Springer-Verlag, Berlin, Germany.

1988. Reafferent control of optomotor yaw torque in Drosophila melanogaster. J. Comp. Physiol. A 163: 373-388.

1993. The sensory-motor link in motion-dependent flight control of flies. In Visual motion and its role in the stabilization of gaze. (ed. F.A. Miles and J. Wallmann), pp. 265-282. Elsevier, Amsterdam, Netherlands.

Hernandez de Salomon, C. and H.Ch. Spatz. 1983. Colour vision in Drosophila melanogaster. Wavelength discrimination. J. Comp. Physiol. A 150: 31-33.

Menne, D. and H.Ch. Spatz. 1977. Color vision in Drosophila melanogaster. J. Comp. Physiol. A 114: 301-312.

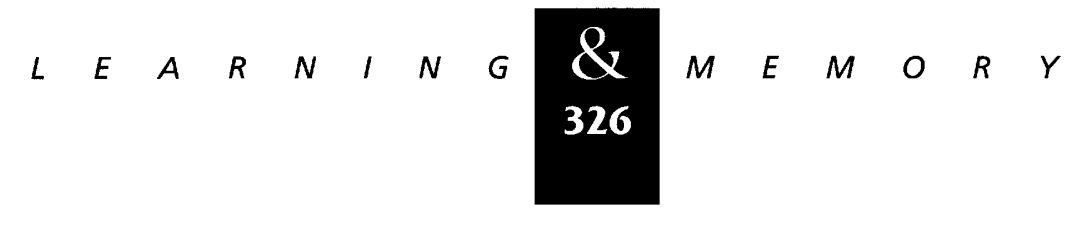


Menzel, R., K. Geiger, L. Chittka, J. Joerges, J. Kunze, and U. Müller. 1996. The knowledge base of bee navigation. J. Exp. Biol. 199: 141-146.

Müller, M. and R. Wehner. 1988. Path integration in desert ants, Cataglyphis fortis. Proc. Natl. Acad. Sci.

85: $5287-5290$.

Reichardt, W. 1973. Musterinduzierte Flugorientierung der Fliege Musca domestica. Naturwissenschaften 60: 122-138.

Reichardt, W. and T. Poggio. 1976. Visual control of orientation behaviour in the fly. Part I. A quantitative analysis. Q. Rev. Biophys. 9: 311-375.

Wehner, R., B. Michel, and P. Antonsen. 1996. Visual navigation in insects: Coupling of egocentric and geocentric information. J. of Exp. Biol. 199: 129-140.

Wolf, R. and M. Heisenberg. 1991. Basic organization of operant behavior as revealed in Drosophila flight orientation. J. Comp. Physiol. A 169: 699-705.

Received July 28, 1997; accepted in revised form October 23, 1997. 


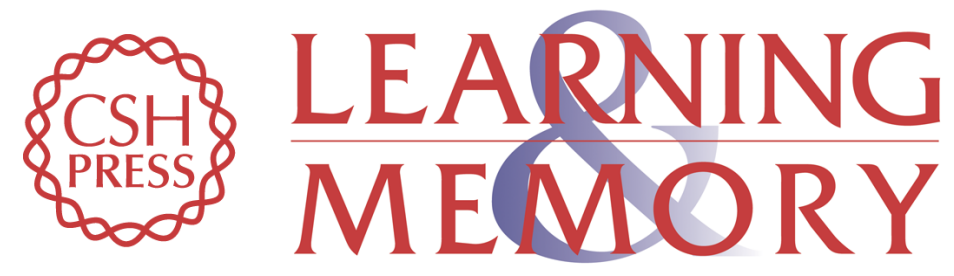

\section{Visual space from visual motion: turn integration in tethered flying Drosophila.}

$\mathrm{R}$ Wolf and $\mathrm{M}$ Heisenberg

Learn. Mem. 1997, 4:

Access the most recent version at doi:10.1101//m.4.4.318

References This article cites 14 articles, 3 of which can be accessed free at: http://learnmem.cshlp.org/content/4/4/318.full.html\#ref-list-1

License

Email Alerting Receive free email alerts when new articles cite this article - sign up in the box at the Service top right corner of the article or click here. 